ARTICLE

Received 2 Mar 2015 | Accepted 2 Jun 2015 | Published 10 Jul 2015 DOI: 10.1038/ncomms8698 OPEN

\title{
Carbon-doped BN nanosheets for metal-free photoredox catalysis
}

Caijin Huang ${ }^{1}$, Cheng Chen ${ }^{1}$, Mingwen Zhang ${ }^{1}$, Lihua Lin ${ }^{1}$, Xinxin $\mathrm{Ye}^{1}$, Sen Lin ${ }^{1}$, Markus Antonietti ${ }^{2}$

$\&$ Xinchen Wang ${ }^{1}$

The generation of sustainable and stable semiconductors for solar energy conversion by photoredox catalysis, for example, light-induced water splitting and carbon dioxide reduction, is a key challenge of modern materials chemistry. Here we present a simple synthesis of a ternary semiconductor, boron carbon nitride, and show that it can catalyse hydrogen or oxygen evolution from water as well as carbon dioxide reduction under visible light illumination. The ternary B-C-N alloy features a delocalized two-dimensional electron system with $s p^{2}$ carbon incorporated in the $h$-BN lattice where the bandgap can be adjusted by the amount of incorporated carbon to produce unique functions. Such sustainable photocatalysts made of lightweight elements facilitate the innovative construction of photoredox cascades to utilize solar energy for chemical conversion.

\footnotetext{
${ }^{1}$ State Key Laboratory of Photocatalysis on Energy and Environment, College of Chemistry, Fuzhou University, Fuzhou 350002, China. ${ }^{2}$ Department of Colloid Chemistry, Max Planck Institute of Colloids and Interfaces, Potsdam 14476, Germany. Correspondence and requests for materials should be addressed to X.W. (email: xcwang@fzu.edu.cn).
} 
T he use of abundant sunlight to split water into $\mathrm{H}_{2}$ and $\mathrm{O}_{2}$ has been considered as the simplest pathway to make clean fuels that can be converted to electricity, for example, in $\mathrm{H}_{2}-\mathrm{O}_{2}$ fuel cells ${ }^{1,2}$. Since the report on photocatalytic water splitting in 1972 (ref. 3), a quantum yield of $\sim 56 \%$ has been achieved for $\mathrm{NiO} / \mathrm{NaTaO}_{3}$ - $\mathrm{La}$ at $270 \mathrm{~nm}$ for overall water splitting ${ }^{4}$, while for visible light systems a highest quantum yield of $\sim 5 \%$ at $420 \mathrm{~nm}$ was reported for $\mathrm{RhCrO} / \mathrm{ZnGaON}^{5}$. These studied promise solar fuel production by photocatalysis. Further progress, however, relies on breakthroughs in the development of sustainable photocatalysts and co-catalysts, classically based on metals 6 , (for example, metal oxides, (oxy)nitride and (oxy)sulfides) for light harvesting, together with noble metal co-catalysts (for example, $\mathrm{Pt}$ and $\mathrm{RuO}_{2}$ ) for driving the two half reactions, water reduction and water oxidation. The two half reactions can be later combined to construct two-photon (Z-scheme) photocatalytic systems to achieve overall water splitting in a cascade fashion ${ }^{8}$, as inspired by the photosynthetic reactions in plants.

Recently, a class of metal-free photocatalysts has been emerged, including elemental boron ${ }^{9}$, sulfur ${ }^{10}$ and phosphor ${ }^{11}$, as well as the binary carbon nitride ${ }^{12}$ and boron carbide ${ }^{13}$. These papers indicate that this construction of stable photocatalysts from lightweight and abundant elements is possible and opens up new opportunities for photochemistry. The two-dimensional (2D) character of their electron systems gives further access to partly 'exotic' physical properties, as described for graphene ${ }^{14}$ and layered hexagonal $\mathrm{BN}(h-\mathrm{BN})^{15-17}$. Graphene, the single atomic layer of carbons expresses a zero bandgap, whereas the $h$-BN possesses a wide bandgap $(\sim 5.5 \mathrm{eV})$. Both materials are therefore, as photocatalyst, not suitable, but intermediates of these layered materials (called ternary BCN compounds) could constitute the desired medium-bandgap semiconductors where bandgap and absolute energy levels can be adjusted by chemical variations ${ }^{18,19}$

Here we present a facile synthesis method to produce a ternary structure of $\mathrm{BCN}$ nanosheets that has a functionality to catalyse hydrogen and oxygen evolution from water as well as $\mathrm{CO}_{2}$ reduction under visible light illumination.

\section{Results}

Theoretical calculations. From density functional theory (DFT) calculations, pure $h$-BN possesses an indirect bandgap of $4.56 \mathrm{eV}$ (Fig. 1a-c), consistent with the previous theoretical values ${ }^{20}$. This is about $1.00 \mathrm{eV}$ smaller than the experimental value ${ }^{21}$, due to the well-known limitation of the DFT within GGA method. As shown in Fig. $1 \mathrm{~g}$ for a ' $\mathrm{B}_{11} \mathrm{C}_{12} \mathrm{~N}_{9}$ '-compound, the gap is significantly reduced from 4.56 to $2.00 \mathrm{eV}$. Different from that of pure $h$-BN (Fig. 1c), the partial density of states indicates that the valence band and conduction band edges of $\mathrm{B}_{11} \mathrm{C}_{12} \mathrm{~N}_{9}$ are mainly composed of the C $2 p$ orbitals (Fig. 1i). Contrary to the localized valence band top of pure $h$-BN, the valence band top states of $\mathrm{B}_{11} \mathrm{C}_{12} \mathrm{~N}_{9}$ are more delocalized as displayed in Fig. $1 \mathrm{~h}$. Calculation results on the B-C-N alloy with a different composition of ' $\mathrm{B}_{13} \mathrm{C}_{8} \mathrm{~N}_{11}$ ' are displayed in Fig. 1e,f, and their computational optical absorption was displayed in Supplementary Fig. 1. Similar results on band structure were attained, but with an enlarged gap of $2.90 \mathrm{eV}$ due to the reduced carbon content in $h$-BN. Clearly, the doping of aromatic carbon into the $h$-BN lattice narrows its bandgap, making it possible to induce visible light catalysis ${ }^{20,22}$.

Preparation of BCN-x samples. Doping of graphene with boron and nitrogen is generally performed to open up its bandgap ${ }^{23}$, but it is still challenging to keep this process sufficiently controlled. a
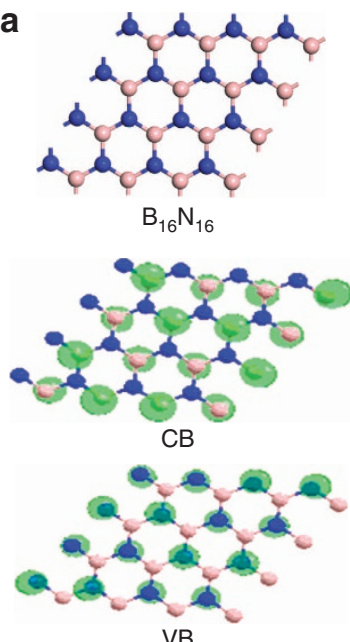

d
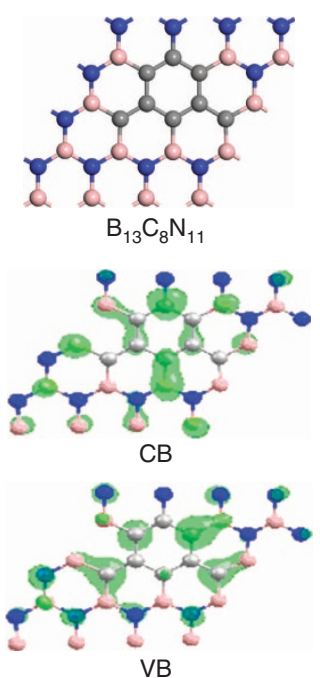

g
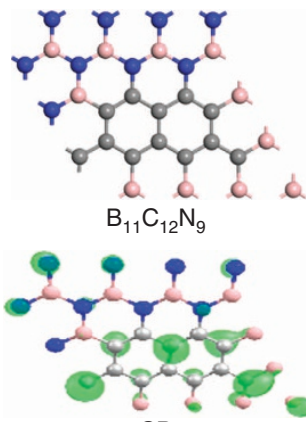

$\mathrm{CB}$

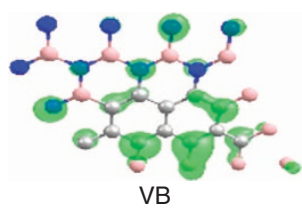

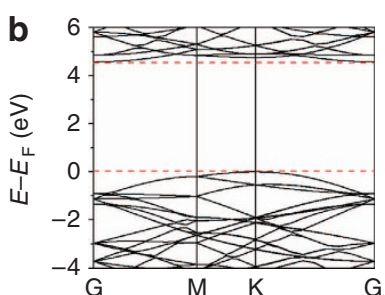

C
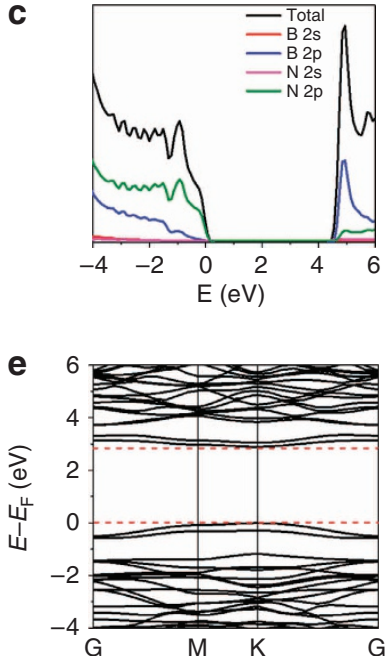

f
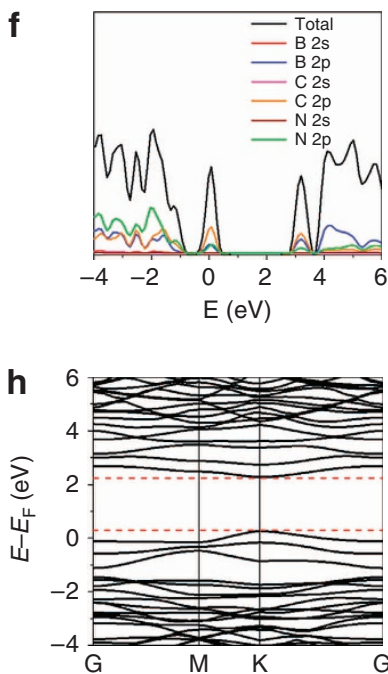

i

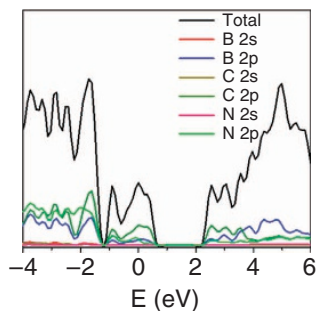

Figure 1 | The plane-wave DFT calculations of electronic structure of $\boldsymbol{h}$-BN and $\boldsymbol{h}$-BCN. The optimized structure of $\mathrm{B}_{16} \mathrm{~N}_{16}$ (a) $\mathrm{B}_{13} \mathrm{C}_{8} \mathrm{~N}_{11}$ (d) and $\mathrm{B}_{11} \mathrm{C}_{12} \mathrm{~N}_{9}$ (g) with the corresponding valence band $(\mathrm{VB}) /$ conduction band $(\mathrm{CB})$, calculated energy band (b,e,h) and corresponding total and ion-decomposed electronic density of states $(\mathbf{c}, \mathbf{f}, \mathbf{i})$.

The adjustment of the carbon doping within $h$ - $\mathrm{BN}^{18}$ seems to us the more easy access to $\mathrm{BCN}$-based materials with high yield in a controllable manner. Herein, we develop such a carbon-doping 
strategy by using a pyrolysis method. In brief, different amounts of glucose were mixed with boron oxide and urea. The mixture was put into a tube furnace in an ammonia atmosphere for $5 \mathrm{~h}$ at $1,250^{\circ} \mathrm{C}$ (see Methods section for details). The obtained samples were named as BCN-x, where $x$ is the glucose weight in per cent of boron oxide.

Characterization of BCN-x samples. Figure 2a shows the X-ray diffraction (XRD) patterns of the prepared samples with different amounts of glucose. Without glucose, the synthesis yielded $h$-BN showing two characteristic XRD peaks at $\sim 26^{\circ}$ and $\sim 43^{\circ}$, attributable to the (002) and (100) planes of the graphitic structure of $h$-BN, respectively ${ }^{24}$. With glucose, all samples featured XRD peaks similar to those of $h$-BN, while increasing
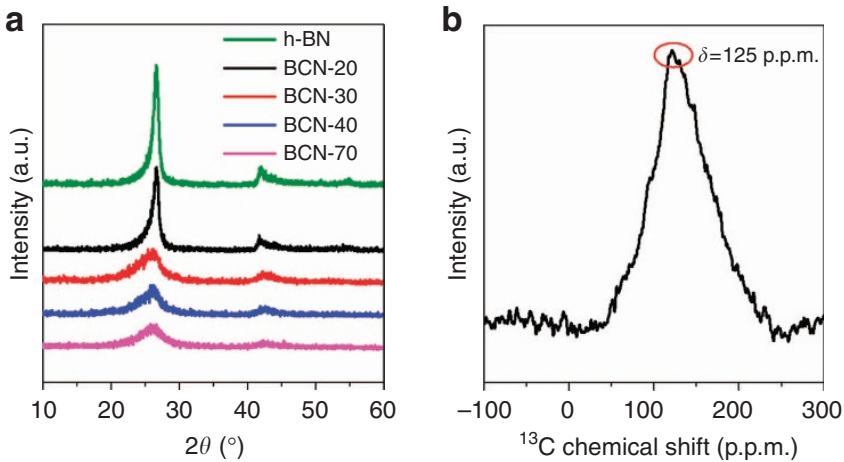

c

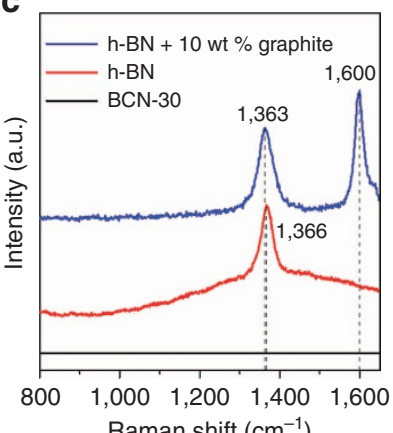

d
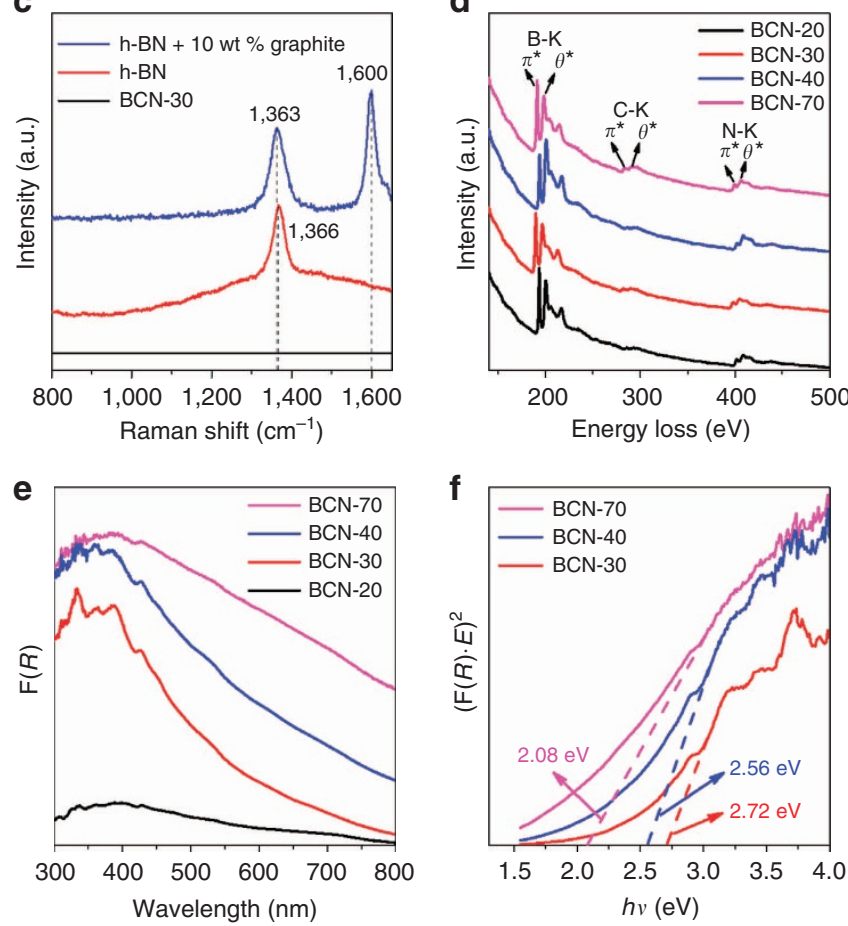

Figure 2 | Structure and optical characterization of $\boldsymbol{h}-\mathrm{BCN}$. (a) Powder XRD patterns of $h-B C N$ synthesized with different amount of glucose, (b) solid-state ${ }^{13} \mathrm{C}$ NMR spectrum of $\mathrm{BCN}-30$, (c) Raman spectra of the $h-\mathrm{BN}, \mathrm{BCN}-30$ and the physical mixture of $h-\mathrm{BN}$ and graphite, (d) EELS spectra of $\mathrm{BCN}-x$ sample, (e) ultraviolet-visible diffuse reflectance spectra (UV-vis DRS) of the BCN-x samples and (f) bandgap determination of the $B C N-x$ samples from the $(F(R) \cdot E)^{n}$ versus $E$ plots. According to the result of theoretical calculation of $h-\mathrm{BCN}$ (Fig. 1g,k), the band structure of $h-\mathrm{BCN}$ is the direct gaps, so the ' $n$ ' is equal to 2 . amounts of glucose broadens the peaks $\mathrm{s}^{25}$, accompanied by a slight shift of the peak to lower angles. This indicates the doping of carbon in the $h$-BN lattice, forming ternary $\mathrm{B}-\mathrm{C}-\mathrm{N}$ alloys.

The chemical structure was further characterized with Fourier transform infrared (FT-IR; Supplementary Fig. 2). Two bands observed at 1,380 and $780 \mathrm{~cm}^{-1}$ correspond to the in-plan B-N transverse stretching vibration and the out-of-plan B-N-B bending vibration, respectively ${ }^{26}$. We failed to detect the $\mathrm{C}-\mathrm{N}$ bonds, which typically overlap with $\mathrm{B}-\mathrm{N}$ bands around $1,100-1,300 \mathrm{~cm}^{-1} \cdot{ }^{13} \mathrm{C}$ solid-state NMR was therefore performed to show the presence of carbon (Fig. 2b). Interestingly, the resonance appears around 125 p.p.m., similar to that of reduced graphene oxide/graphite ${ }^{27}$, which corresponds to $s p^{2}$ carbons with an electron density very similar to graphite. This means that in this $\mathrm{BCN}$ alloy the influence of the electronegativity of $\mathrm{N}$ and electropositivity of B balance each other out, with the carbon as such being only slightly more electron rich than in graphite. The incorporation of carbon in the $h$-BN layers smears out Raman signals of $h$-BN due to the distortion of the layer symmetry ${ }^{28}$ (Fig. 2c). Note that the Raman peaks of $h$-BN remain for the physical mixture of $h$ - $\mathrm{BN}$ and graphite, measured as a reference.

Supplementary Fig. 3 is the X-ray photoelectron spectroscopic (XPS) spectra of the BCN product. The main peak of B1s at $190.4 \mathrm{eV}$ is attributed to $\mathrm{B}$ atoms surrounded by $\mathrm{N}$ atoms ${ }^{29}$. The subpeaks at 189.9 and $192.3 \mathrm{eV}$ are due to the $\mathrm{B}-\mathrm{C}$ band and unreacted $\mathrm{B}-\mathrm{O}$, the latter potentially being a surface impurity ${ }^{25}$. The $\mathrm{C} 1 \mathrm{~s}$ signal at $284.6 \mathrm{eV}$ is due to the graphitic carbon $(\mathrm{C}=\mathrm{C})$, while the peaks at 285.7 and $283.5 \mathrm{eV}$ are assigned to $\mathrm{C}-\mathrm{N}$ bonds and $\mathrm{C}-\mathrm{B}$ bonds, respectively ${ }^{29}$. The corresponding $\mathrm{N}$-signals for $\mathrm{N}-\mathrm{B}$ or $\mathrm{N}-\mathrm{C}$ bands are also observed and located at 397.9 and $398.6 \mathrm{eV}$ (ref. 29). The small subpeak at $400.5 \mathrm{eV}$ reflects $\mathrm{N}-\mathrm{H}$ bonds, again presumably needed for edge termination and reflecting the reductive ammonia atmosphere. Elemental analysis by XPS (Supplementary Table 1) revealed that the carbon content is gradually increasing with increasing amounts of glucose. Thus, XPS analysis very clearly supports the incorporation of carbon in the $h$-BN.

Figure $2 \mathrm{~d}$ displays the electron energy loss spectroscopy (EELS) of the $h$-BCN samples, showing the K-edges of $\mathrm{B}, \mathrm{C}$ and $\mathrm{N}$. The $\mathrm{B}$ - and $\mathrm{N}$-signal of $\mathrm{BCN}$ coincide with those of $h$ - $\mathrm{BN}$, reflecting a similar incorporation in layered $s p^{2}$ domains ${ }^{18,23}$. The sharp doublet $\mathrm{C}-\mathrm{K}\left(\pi^{*}\right)$ and $\mathrm{C}-\mathrm{K}\left(\theta^{*}\right)$ evidence a rather perfect $s p^{2}$-bonding neighbourhood also for the carbon positions. In addition, the carbon doping actually commutatively affects the corresponding $\mathrm{B}-\mathrm{K}, \mathrm{N}-\mathrm{K}$ and $\mathrm{C}-\mathrm{K}$ energies. All data indicate that $\mathrm{B}, \mathrm{C}$ and $\mathrm{N}$ are in a commonly $s p^{2}$-hybridized, $2 \mathrm{D}$-conjugated electron system, rather than a physical mixture.

Figure 3 depicts transmission electron microscopy (TEM) and STEM images of the BCN-30, and the scanning electron microscopy and energy-dispersive X-ray spectroscopy analyses were presented in Supplementary Fig. 4. A flake-like morphology similar to few-layer-graphene gives evidence that folded BCN layers constitute the structure. High-resolution TEM (inset of Fig. 3a) allows the observation of an interlayer crystal lattice spacing of $3.40 \AA$. The nanosheets are composed of $\sim 10$ stacked layers and have $3-4 \mathrm{~nm}$ overall thickness. Such $2 \mathrm{D}$ systems with thicknesses below $10 \mathrm{~nm}$ are particularly interesting for optoelectrics as the exciton and charge diffusion length is generally in that size range ${ }^{30}$. The selected-area electron diffraction (inset of Supplementary Fig. 5) also suggests that the in-planar order of the sample is polycrystalline, which might reflect that the carbon forms a solid solution or constitutes grain boundaries of $\mathrm{BN}$ nanodomains. Element mapping of $\mathrm{BCN}-30$ (Fig. 3b) gave a uniform distribution of $\mathrm{B}, \mathrm{C}$ and $\mathrm{N}$ throughout the whole selected area, proving homogeneity of the ternary $\mathrm{BCN}$ alloys. This is also proven by the STEM image that clearly displays homogeneous 

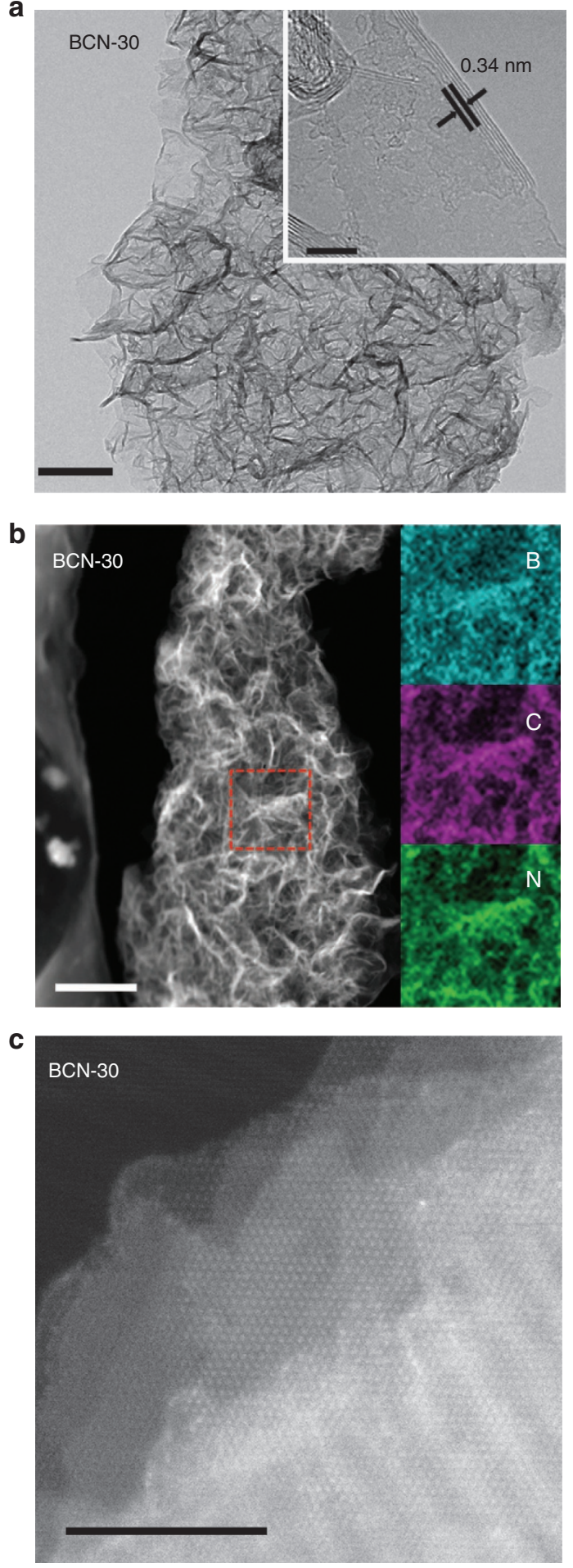

Figure 3 | Surface morphology of $\mathbf{B C N}-\mathbf{3 0}$. (a) HRTEM image of the $\mathrm{BCN}-30$ sample. Scale bar, $100 \mathrm{~nm}$, inset scale bars, $5 \mathrm{~nm}$. (b) Typical TEM dark-field image of $\mathrm{BCN}-30$ sample and the elemental mapping images of $\mathrm{B}$, $\mathrm{C}$ and $\mathrm{N}$ of the enlargement of selected-area in the picture. Scale bar, $300 \mathrm{~nm}$. (c) High-resolution STEM of the BCN-30 sample along (002) facet. Scale bar, $5 \mathrm{~nm}$.

mixing of atoms in the (002) facet (Fig. 3c), without finding grain boundaries. The ternary $\mathrm{BCN}$ is rather stable up to $800^{\circ} \mathrm{C}$ in the air, even (Supplementary Fig. 6).
The optical absorption of the samples is shown in Fig. 2e. Carbon-free $h$-BN made as such is a typical insulator with a bandgap of about $5.6 \mathrm{eV}$ (refs 16,21). As shown in Fig. 2f, the optical absorption edge of BCN alloys gradually red-shifts as the amount of carbon increased. From the plot of the transformed Kubelka-Munk function versus light energy, a bandgap of $2.72 \mathrm{eV}$ was estimated for BCN-30. The long absorption tails in the visible however indicates the presence of intraband impurity transitions, potentially located at the surface. Further optimization of the synthesis of high-quality $h$-BCN alloys is certainly a valuable option. Nevertheless, for a proof-of-concept advance, the optical properties are sufficient to generate electron-hole pairs on $h$-BCN as confirmed by photoelectrochemical (Supplementary Fig. 7) and electron paramagnetic resonance characterizations (Supplementary Fig. 8a,b). The band positions of the $h$-BCN is estimated to straddle the water redox potentials, which are however tuneable by the amount of carbon doped (Supplementary Fig. 9). Therefore, we could apply these new semiconductors for the first time for visible light photocatalysis.

\section{Discussion}

The photocatalytic performance of BCN samples was assessed by a $\mathrm{H}_{2}$ evolution assay. $\mathrm{H}_{2}$ evolution under light was found, while in dark the samples are inactive. The best sample is BCN-30, while hydrogen evolution rate gradually decreases as the carbon content further increases (Supplementary Fig. 10). Excessive amounts of carbon presumably diminish the size of ordered domains and weaken the semiconductor properties, as confirmed by the decrease in the charge-carrier lifetime with excessive carbon doped (Supplementary Fig. 11). It is however a remarkable observation that the $\mathrm{BCN}$ alloys could photocatalyse hydrogen generation even without using noble metals as a cocatalyst and even with visible light. Under those conditions, the samples present a quite stable activity (Fig. 4a), but a deactivation was observed when the materials was illuminated with ultraviolet light (Supplementary Fig. 12a).

We also modified BCN with $\mathrm{Pt}$ for $\mathrm{H}_{2}$ evolution, but observed only comparable $\mathrm{H}_{2}$ production in the range of $0.5-3 \mathrm{wt} \% \mathrm{Pt}$ (Supplementary Fig. 13). This indicates that the surface of BCN alloys are already rather active, and one can speculate about a pro forma hydride elimination from the boron sites (corresponds to a reversible hydrogenation of $\mathrm{a}-\mathrm{N}=\mathrm{B}$-bond) in the catalytic cycle. However, the stability of BCN under strong ultraviolet irradiation is remarkably increased by adding Pt (Supplementary Fig. 12b). We can speculate that the $\mathrm{Pt}$ is avoiding the over-reduction of the $-\mathrm{N}=\mathrm{B}$-structure and the consecutive reductive disintegration of the material. The overall production of hydrogen gas amounts to $4,570 \mu \mathrm{mol}$ in these two-cycle reactions, exceeding the amount of $h$-BCN $(1,342 \mu \mathrm{mol})$ and Pt $(5.12 \mu \mathrm{mol})$. After the reaction, the structure of BCN-30 was stable as reflected by the XRD, FTIR and TEM examinations of the used photocatalyst (Supplementary Fig. 14). The apparent quantum efficiency of BCN-30 was calculated to be $0.54 \%$ at $405 \mathrm{~nm}$ (Supplementary Table 2) by using equation (1). We also compared the performance of BCN-30 with other photocatalysts, and results show that the activity of BCN-30 is higher than that of $\mathrm{TiO}_{2}$ (P25) and $g-\mathrm{C}_{3} \mathrm{~N}_{4}$ under visible light (Supplementary Table 3 ). It is noted that there is not clear relationship between the specific surface area and hydrogen evolution rate of $\mathrm{BCN}$ samples (Supplementary Table 4). This is due to the fact that in most solid-liquid phase photocatalysis the reaction rate is basically limited by charge separation instead of mass transfer.

The $\mathrm{H}_{2}$ generation from water is pleasing, but it constitutes only the minor half of the problem of water splitting. The other half-reaction, the oxygen evolution reaction, is particularly 

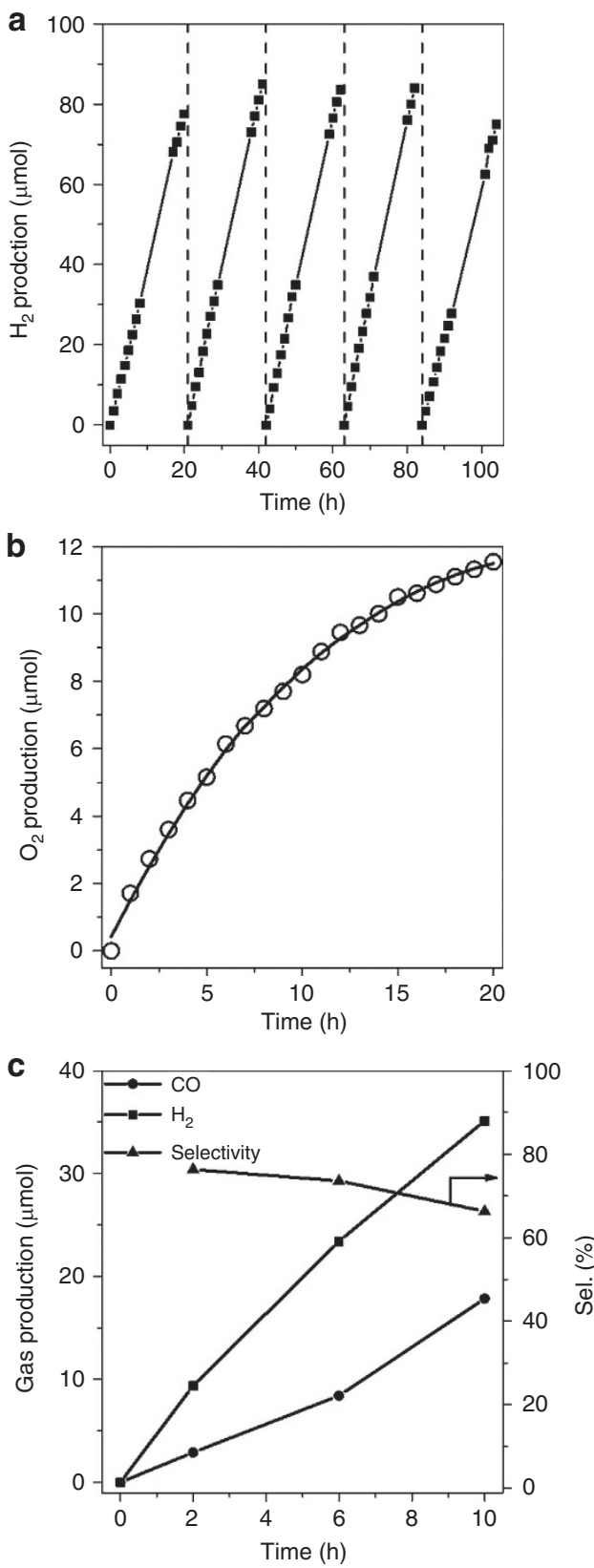

Figure 4 | Photocatalytic performance of BCN-30. (a) Stable hydrogen evolution from water by $\mathrm{BCN}-30$ under visible light. The reaction was continued for $104 \mathrm{~h}$, with evacuation every $20 \mathrm{~h}$ (dashed line). (b) Time courses of oxygen production from water by $\mathrm{Ni}-\mathrm{Co} \mathrm{LDH} / \mathrm{BCN}-30$ under visible light illumination $(\lambda>420 \mathrm{~nm}$ ). (c) Time conversion plot for photocatalytic $\mathrm{CO}_{2}$ reduction. The amount of $\mathrm{CO}$ and $\mathrm{H}_{2}$ produced from the $\mathrm{CO}_{2}$ conversion system as a function of reaction time under visible light illumination $(\lambda>420 \mathrm{~nm})$. Selectivity $=n \mathrm{CO} / n\left(\mathrm{CO}+\mathrm{H}_{2}\right) \times 100 \%$.

challenging because it requires the promotion of four redox processes over a narrow potential range, the coupling of multiple proton and electron transfers, and the formation of an oxygen-oxygen bond. As a first trial to promote oxygen evolution from water by $\mathrm{BCN}$, we choose $\mathrm{RuO}_{2}$ and $\mathrm{IrO}_{3}$, and $\mathrm{Ni}-\mathrm{Co}$ layered double hydroxides (Ni-Co LDHs) as cocatalysts ${ }^{31}$. Results revealed that $\mathrm{Ni}-\mathrm{Co} \mathrm{LDHs}$ is the best cocatalyst (Supplementary Fig. 15), potentially due to intimate interface contacts between the two layered structures. As shown in Fig. $4 \mathrm{~b}$, the Ni-Co LDHs/BCN-30 catalyst liberated $11 \mu \mathrm{mol} \mathrm{O} \mathrm{O}_{2}$ in $20 \mathrm{~h}$ reaction with visible light, and with ultraviolet light the experiment gave $35 \mu \mathrm{mol} \mathrm{O}{ }_{2}$ (Supplementary Fig. 16).

These results indicate that the band positions of $h-\mathrm{BCN}$ are correctly positioned for water splitting, that is, the $\mathrm{Ni}-\mathrm{Co} \mathrm{LDH}$ is able to take up the photogenerated holes and evolves $\mathrm{O}_{2}$. The decrease in activity with reaction time is primarily due to the deposition of metallic silver at the catalyst surface, which blocks light absorption and obstructs active sites ${ }^{12}$. Further optimization of the $\mathrm{O}_{2}$ evolution system is thus certainly needed as the found activity is only moderate.

Having a very reductive photoelectron however allows to address the direct photochemical conversion of $\mathrm{CO}_{2}$ (ref. 32). We therefore expanded in a last experiment the application of $h$-BCN to the photocatalytic reduction of $\mathrm{CO}_{2}$ to $\mathrm{CO}$ (Fig. 4c) using visible light $(\lambda>420 \mathrm{~nm})$. On irradiation for $2 \mathrm{~h}$, the system evolved $\mathrm{CO}(9.3 \mu \mathrm{mol})$ and $\mathrm{H}_{2}(2.9 \mu \mathrm{mol})$. A prolonged operation of the photochemical system gave a gradual increase in both $\mathrm{CO}$ and $\mathrm{H}_{2}$ evolutions in a linear fashion. Isotopic experiments confirmed the carbon source of the produced ${ }^{13} \mathrm{CO}$ is ${ }^{13} \mathrm{CO}_{2}$ (Supplementary Fig. 17).

In summary, we introduced here a novel and simple way of carbon doping of $h$-BN nanosheets to generate ternary BCN alloys as sustainable and stable visible light photocatalysts. Water splitting in the elements as well as $\mathrm{CO}_{2}$ reduction was proven to be possible. This new photocatalyst features a $2 \mathrm{D}$ electron system and tuneable bulk and surface properties, and further structural optimizations can be expected that may facilitate innovations and applications in the fields from artificial photosynthesis to graphene-like semiconductors, as well as organocatalysis.

\section{Methods}

Synthesis of BCN-x. Typically, boron oxide $(2 \mathrm{~g})$, urea $(4 \mathrm{~g})$ and a certain amount of glucose were grinded fully with an agate mortar. After that, mixed precursor was put into a horizontal tube furnace. Before heating up, it costs nearly $30 \mathrm{~min}$ to expel the whole oxygen in the tube and the sample was then heated to $1,250{ }^{\circ} \mathrm{C}$ for $5 \mathrm{~h}$ The obtained products were washed with $0.1 \mathrm{M} \mathrm{HCl}$ in hot water. The resulting final sample was denoted as $\mathrm{BCN}-x$, where $x(20,30,40,70)$ is the percentage weight content of glucose to boron oxide. When the glucose was omitted, the synthesized sample was named $h$-BN.

Synthesis of Ni-Co LDHs/BCN-30. $\mathrm{Co}(\mathrm{NO})_{2} \cdot 6 \mathrm{H}_{2} \mathrm{O}(0.2 \mathrm{~g}), \mathrm{Ni}\left(\mathrm{NO}_{3}\right)_{2} \cdot 6 \mathrm{H}_{2} \mathrm{O}$ $(0.1 \mathrm{~g})$ and $\mathrm{NH}_{4} \mathrm{NO}_{3}(0.04 \mathrm{~g})$ were dissolved in $\mathrm{H}_{2} \mathrm{O}(3.5 \mathrm{ml})$ and $30 \mathrm{wt} \%$ ammonia $(1.5 \mathrm{ml})$ to form clear solution, named as Ni-Co LDHs. $200 \mathrm{mg}$ BCN-30 powder was dispersed in water $(20 \mathrm{ml})$, which was then subject to ultrasonic treatment for $10 \mathrm{~min}$ to promote the dispersion of $\mathrm{BCN}-30$ in the solution. A certain amount of the Ni-Co LDHs solution $(400 \mu \mathrm{l})$ was added to the mixture. After stirring for $3 \mathrm{~h}$, the mixture was washed by filtering with distilled water and dried at $343 \mathrm{~K}$ to obtain Ni-Co LDHs/BCN-30.

Characterization. Powder XRD patterns were collected on Bruker D8 Advance diffractometer with $\mathrm{Cu}-\mathrm{K} 1$ radiation $(\lambda=1.5406 \AA)$. Data were collected with a rate of $0.02^{\circ} / 2 \theta$ in the range of 10 to $60^{\circ}$. The FT-IR spectra were obtained on a Nicolet 670 FT-IR spectrometer with $\mathrm{KBr}$ as the diluents. To get smooth spectra, the final results were registered after accumulation of 108 scans and a resolution of $4 \mathrm{~cm}^{-1}$ XPS measurements were performed on an ESCALAB 250 (Thermo Scientific, USA) by using a monochromatized $\mathrm{Al} \mathrm{K} \alpha$ line source $(200 \mathrm{~W})$. Solid-state ${ }^{13} \mathrm{C}$ MAS NMR experiments were conducted on a spectrometer (Bruker Avance III-400WB) equipped with a $4-\mathrm{mm} \mathrm{ZrO}_{2}$ rotor probe operating at a $5 \mathrm{kHz}$ spinning rate for ${ }^{13} \mathrm{C}$ nuclei. The direct ${ }^{13} \mathrm{C}$-polarization spectrum was acquired with a $90^{\circ}$ pulse of $5 \mu \mathrm{s}$ for a pulse repetition delay of $2 \mathrm{~s}$ for 2,048 scans. The ${ }^{13} \mathrm{C}$ spectrum was referenced to tetramethylsilane at 0 p.p.m. using adamantane as an external reference. Raman spectroscopic measurements were performed on a Renishaw in Via Raman System 1000 with a $532 \mathrm{~nm} \mathrm{Nd:YAG} \mathrm{excitation} \mathrm{source} \mathrm{at} \mathrm{room} \mathrm{temperature.} \mathrm{The} \mathrm{UV/Vis}$ picture was recorded on a Cary 500 Scan Spectrophotometer (Varian, USA). Here $\mathrm{BaSO}_{4}$ was used as a reflectance standard in the ultraviolet-visible diffuse reflectance experiment. Nitrogen adsorption-desorption isotherms were collected at $77 \mathrm{~K}$ using a Micromeritics ASAP 2020 surface area and porosity analyser. Thermogravimetric analysis was performed on TG209 (NETZSCH Co.). Photoluminescence spectra were recorded on an Edinburgh FI/FSTCSPC 920 spectrophotometer. The morphology of the sample and energy-dispersive X-ray spectroscopy were investigated by Hitachi S4800 field emission scanning electron microscopy. TEM was operated by Tecnai20 FEG microscope. The elemental 
mapping, and selected-area electron diffraction were also collected on the TEM machine. High-resolution STEM and EELS investigation were carried out on a JEM-ARM200CF microscope (JEOL, Tokyo, Japan) with double CS correctors for the condenser lens and objective lens at an operating voltage of $80 \mathrm{kV}$. HAADF images were acquired at acceptance angles of 70-150 mrad. Photoelectrochemical analysis was conducted with a BAS Epsilon Electrochemical System in a three electrode cell, using an $\mathrm{Ag} / \mathrm{AgCl}$ electrode $(3 \mathrm{M} \mathrm{KCl})$ as the reference electrode and a Pt plate as the counter electrode. The working electrode was prepared by spreading with catalyst slurry $\left(5 \mathrm{mg} \mathrm{ml}^{-1}\right.$ in DMF) on indium-tin oxide. After air drying, the working electrode was put into a $0.2-\mathrm{M} \mathrm{Na}_{2} \mathrm{SO}_{4}$ aqueous solution $(\mathrm{pH}=6.8)$. Electron paramagnetic resonance analysis was conducted with $\mathrm{A}$ Bruker A300 spectrometer, with the following settings: centre field, $3512.48 \mathrm{G}$; microwave frequency, $9.86 \mathrm{GHz}$; and power, $6.35 \mathrm{~mW}$.

Theoretical calculation. The plane-wave DFT calculations were carried out by using the Vienna $a b$ initio simulation package with the gradient-corrected PW91 exchange-correction function. For valence electrons, a plane-wave basis set was adopted with an energy cutoff of $400 \mathrm{eV}$ and the ionic cores were described with the projector augmented-wave method. The optimized lattice parameter for a $h$-BN monolayer was calculated to be $2.49 \AA$, which is in good agreement with the experimental result $(2.54 \AA)^{33}$. A $4 \times 4$ supercell $h$-BN containing 32 atoms of $h$-BN is constructed as a substrate. For the optimization of all the relaxation atoms, $3 \times 3 \times 1$ Monkhorst-Pack $k$-point grids were used to sample the Brillouin zone, which were tested to be converged, whereas for electronic properties calculations, the Brilliouin zone was sampled by $9 \times 9 \times 1 k$-points. In addition, to avoid the interlayer interactions, a vacuum spacing in the $z$ direction was set to be $14 \AA$. Here the possible structure of the $\mathrm{BCN}$ was constructed based on our experimental section. At present, there is a great controversy of the BCN structure. Some argue that the $\mathrm{BN}$ and $\mathrm{C}$ present independent just like a carbon island or $\mathrm{BN}$ island while other think the $\mathrm{C}$ is a part of the $\mathrm{BN}$, which means that there is a $\mathrm{C}-\mathrm{B}$ or $\mathrm{C}-\mathrm{N}$ bond in the compound. In our synthetic section, it possessed $5 \mathrm{~h}$ to reaction under high temperature. Maybe in the early reaction section, the $\mathrm{C}, \mathrm{B}, \mathrm{N}$ can group freely, but later the compound will reform to a lower free energy compound. Previous theory and experiment results have already testified that the bonding energy of $\mathrm{C}-\mathrm{C}$ and $\mathrm{B}-\mathrm{N}$ bonds is comparatively higher than that of other hetero bonds present in the ternary system, consequently, the hybrid systems will be domain-segregated into two different regions, one with C-rich domain and other with BN-enriched domain, to gain thermodynamic stability. Fortunately, our results are coincided with this common sense. So, we build the possible structures like above.

Photocatalytic reaction for water reduction and oxidation. Reactions were experimented with a Pyrex top irradiation reactor connected to a glass closed gas-circulation system. Photoreduction to $\mathrm{H}_{2}$ and photooxidation to $\mathrm{O}_{2}$ were performed separately in aqueous solutions containing triethanolamine or silver nitrate as sacrificial reagents, respectively. To study the $\mathrm{H}_{2}$ production ability, $50 \mathrm{mg}$ catalyst was dispersed into $100 \mathrm{ml}$ aqueous containing triethanolamine solution (10 vol\%). In the case of deposition of $\mathrm{Pt}$, an appropriate amount of $\mathrm{H}_{2} \mathrm{PtCl}_{6}$ aqueous was added to the reactant solution before irradiation. Water photooxidation was carried out by dispersing the catalyst $(50 \mathrm{mg})$ in $100 \mathrm{ml}$ aqueous solution containing silver nitrate $(0.01 \mathrm{M})$ as the electron acceptor and $\mathrm{La}_{2} \mathrm{O}_{3}(0.2 \mathrm{~g})$ as the $\mathrm{pH}$ buffer agent $(\mathrm{pH} 8-9)$. The reactant solution was evacuated several times to remove air completely before irradiation under a $300 \mathrm{~W}$ xenon lamp with an appropriate cutoff filter. The temperature of the solution was maintained at $12{ }^{\circ} \mathrm{C}$ by a flow of cooling water during the reaction. The evolved gases were analysed by a SHIMADZU GC-8 A gas chromatograph with the thermal conductive detector, a 5- $\AA$ molecular sieve column and using Argon as the carrier gas.

The apparent quantum efficiency (AQE) for $\mathrm{H}_{2}$ evolution was performed in the same experiment set-up but with a $405 \mathrm{~nm}$ diode laser as incident light source. The irradiation area was $7.92 \mathrm{~cm}^{2}$. The total intensity of irradiation was determined by averaging 40 points of the irradiation area and the average intensity was estimated to be $4.24 \mathrm{~mW} \mathrm{~cm}^{-2}$ (ILT 950 spectroradiometer). The AQE was calculated as follow:

$$
\mathrm{AQE}=\frac{N_{\mathrm{e}}}{N_{\mathrm{p}}} \times 100 \%=\frac{2 \times M \times N_{\mathrm{A}} \times h \times c}{S \times P \times t \times \lambda} \times 100 \%
$$

Where, $N_{\mathrm{p}}$ is the total incident photons, $N_{\mathrm{e}}$ is the total reactive electrons, $M$ is the amount of $\mathrm{H}_{2}$ molecules, $N_{\mathrm{A}}$ is Avogadro constant, $h$ is the Planck constant, $c$ is the speed of light, $S$ is the irradiation area, $P$ is the intensity of irradiation light, $t$ is the photoreaction time and $\lambda$ is the wavelength of the monochromatic light.

Photocatalytic reduction of $\mathrm{CO}_{2}$. The test was conducted at atmospheric pressure of $\mathrm{CO}_{2}$ in a two neck flask $(50 \mathrm{ml})$ at $30^{\circ} \mathrm{C}$ as controlled by water with a constant temperature. The photocatalytic $\mathrm{CO}_{2}$ reduction reaction was carried out by dispersing $50 \mathrm{mg}$ catalyst in a solution containing solvent of $2 \mathrm{ml} \mathrm{H}_{2} \mathrm{O}$ and $4 \mathrm{ml}$ acetonitrile, $1 \mathrm{ml}$ triethanolamine, $1 \mu \mathrm{mol} \mathrm{CoCl}_{2}, 20 \mathrm{mg}$ 2, 2-bipyridine. This mixture system was subjected to vacuum degassing and backfilling with pure $\mathrm{CO}_{2}$ gas $(1 \mathrm{bar})$. Then the reaction flask was performed under an irradiation of $300 \mathrm{~W}$ xenon lamp with $420 \mathrm{~nm}$ cutoff filter. The produced gases $\left(\mathrm{CO}\right.$ and $\mathrm{H}_{2}$ ) were detected using a gas chromatography equipped with a packed molecular sieve column (TDX-1 mesh 42/10). Argon was used as the carrier gas.

\section{References}

1. Jacobson, M. Z., Colella, W. G. \& Golden, D. M. Cleaning the air and improving health with hydrogen fuel-cell vehicles. Science 308, 1901-1905 (2005).

2. Lewis, N. S. \& Nocera, D. G. Powering the planet: chemical challenges in solar energy utilization. Proc. Natl Acad. Sci. USA 103, 15729-15735 (2006).

3. Fujishima, A. \& Honda, K. Electrochemical photolysis of water at a semiconductor electrode. Nature 238, 37-38 (1972).

4. Kato, H., Asakura, K. \& Kudo, A. Highly efficient water splitting into $\mathrm{H}_{2}$ and $\mathrm{O}_{2}$ over lanthanum-doped $\mathrm{NaTaO}_{3}$ photocatalysts with high crystallinity and surface nanostructure. J. Am. Chem. Soc. 125, 3082-3089 (2003).

5. Maeda, K. et al. Photocatalyst releasing hydrogen from water. Nature 440, 295-295 (2006).

6. Chen, X., Shen, S., Guo, L. \& Mao, S. S. Semiconductor-based photocatalytic hydrogen generation. Chem. Rev. 110, 6503-6570 (2010).

7. Kudo, A. \& Miseki, Y. Heterogeneous photocatalyst materials for water splitting. Chem. Soc. Rev. 38, 253-278 (2009).

8. Tada, H., Mitsui, T., Kiyonaga, T., Akita, T. \& Tanaka, K. All-solid-state Z-scheme in CdS-Au-TiO2 three-component nanojunction system. Nat. Mater. 5, 782-786 (2006).

9. Liu, G., Yin, L.-C., Niu, P., Jiao, W. \& Cheng, H.-M. Visible-light-responsive $\beta$-Rhombohedral boron photocatalysts. Angew. Chem. Int. Ed. 52, 6242-6245 (2013).

10. Liu, G., Niu, P., Yin, L. \& Cheng, H.-M. $\alpha$-Sulfur crystals as a visible-light-active photocatalyst. J. Am. Chem. Soc. 134, 9070-9073 (2012).

11. Wang, F. et al. Red phosphorus: an elemental photocatalyst for hydrogen formation from water. Appl. Catal. B- Environ. 111-112, 409-414 (2012).

12. Wang, X. et al. A metal-free polymeric photocatalyst for hydrogen production from water under visible light. Nat. Mater. 8, 76-80 (2009).

13. Liu, J. et al. Boron carbides as efficient, metal-free, visible-light-responsive photocatalysts. Angew. Chem. Int. Ed. 52, 3241-3245 (2013).

14. Novoselov, K. S. et al. Electric field effect in atomically thin carbon films. Science 306, 666-669 (2004).

15. Chopra, N. G. et al. Boron nitride nanotubes. Science 269, 966-967 (1995).

16. Watanabe, K., Taniguchi, T. \& Kanda, H. Direct-bandgap properties and evidence for ultraviolet lasing of hexagonal boron nitride single crystal. Nat. Mater. 3, 404-409 (2004).

17. Song, L. et al. Binary and ternary atomic layers built from carbon, boron, and nitrogen. Adv. Mater. 24, 4878-4895 (2012).

18. Ci, L. et al. Atomic layers of hybridized boron nitride and graphene domains. Nat. Mater. 9, 430-435 (2010).

19. Lu, J. et al. Order-disorder transition in a two-dimensional boron-carbonnitride alloy. Nat. Commun. 4, 2681 (2013).

20. Li, X., Zhao, J. \& Yang, J. Semihydrogenated BN sheet: a promising visible-light driven photocatalyst for water splitting. Sci. Rep 3, 1858 (2013).

21. Song, L. et al. Large scale growth and characterization of atomic hexagonal boron nitride layers. Nano Lett. 10, 3209-3215 (2010).

22. Wang, S. et al. BCN graphene as efficient metal-free electrocatalyst for the oxygen reduction reaction. Angew. Chem. Int. Ed. 51, 4209-4212 (2012).

23. Stephan, O. et al. Doping graphitic and carbon nanotube structures with boron and nitrogen. Science 266, 1683-1685 (1994).

24. Shi, Y. et al. Synthesis of few-layer hexagonal boron nitride thin film by chemical vapor deposition. Nano Lett. 10, 4134-4139 (2010).

25. Lei, W., Portehault, D., Dimova, R. \& Antonietti, M. Boron carbon nitride nanostructures from salt melts: tunable water-soluble phosphors. J. Am. Chem. Soc. 133, 7121-7127 (2011).

26. Geick, R., Perry, C. H. \& Rupprecht, G. Normal modes in hexagonal boron nitride. Phys. Rev. 146, 543-547 (1966).

27. Gao, W., Alemany, L. B., Ci, L. \& Ajayan, P. M. New insights into the structure and reduction of graphite oxide. Nat. Chem. 1, 403-408 (2009).

28. $\mathrm{Yu}$, J. et al. Vertically aligned boron nitride nanosheets: chemical vapor synthesis, ultraviolet light emission, and superhydrophobicity. ACS Nano 4, 414-422 (2010)

29. Kim, S. Y. et al. X-ray photoelectron spectroscopy and first principles calculation of BCN nanotubes. J. Am. Chem. Soc. 129, 1705-1716 (2007).

30. Forrest, S. R. The limits to organic photovoltaic cell efficiency. MRS Bull. 30, 28-32 (2005)

31. Hu, Z.-A. et al. Synthesis and electrochemical characterization of mesoporous CoxNil - $\mathrm{x}$ layered double hydroxides as electrode materials for supercapacitors. Electrochim. Acta 54, 2737-2741 (2009).

32. Wigley, T. M. L., Richels, R. \& Edmonds, J. A. Economic and environmental choices in the stabilization of atmospheric $\mathrm{CO} 2$ concentrations. Nature $\mathbf{3 7 9}$, 240-243 (1996).

33. Kurakevych, O. O. \& Solozhenko, V. L. Rhombohedral boron subnitride, $\mathrm{B}_{13} \mathrm{~N}_{2}$ by X-ray powder diffraction. Acta Crystallogr. Sect. C: Cryst. Struct. Commun. 63, i80-i82 (2007) 


\section{Acknowledgements}

This work was supported by the National Basic Research Program of China (973 Program) (2013CB632405), the National Natural Science Foundation of China $(21425309,21203026$ and 21273038), the State Key Laboratory of NBC Protection for Civilian (SKLNBC2013-04K) and the Specialized Research Fund for the Doctoral Program of Higher Education (20133514110003). We appreciate Professor Chen and Dr Zhang for the measurement of photoluminescence lifetime and TEM.

\section{Author contributions}

X.W. conceived and designed the experiments. C.H., C.C. and M.Z. synthesized the experimental samples and carried out the experiments of XRD, NMR, XPS, BET, FT-IR, EPR, Raman, DRS, SEM, EDX, TEM, SAED, TG, photo-current and photocatalytic hydrogen production. M.Z. performed photocatalytic oxygen production experiments. C.C. carried out $\mathrm{CO}_{2}$ reduction experiments. L.L. conducted the experiment of electrochemical analyses and the measurement of the quantum yield of the samples. C.H. and L.L. carried out PL experiment. X.W. and C.H. performed EELS and STEM experiments. S.L. and X.Y. worked on all theoretical calculations. M. A. participated in the scientific discussion on the experimental results. All the authors discussed the results and wrote the manuscript.

\section{Additional information}

Supplementary Information accompanies this paper at http://www.nature.com/ naturecommunications

Competing financial interests: The authors declare no competing financial interests.

Reprints and permission information is available online at http://npg.nature.com/ reprintsandpermissions/

How to cite this article: Huang, C. et al. Carbon-doped BN nanosheets for metal-free photoredox catalysis. Nat. Commun. 6:7698 doi: 10.1038/ncomms8698 (2015).

\section{cc) (i)}

This work is licensed under a Creative Commons Attribution 4.0 International License. The images or other third party material in this article are included in the article's Creative Commons license, unless indicated otherwise in the credit line; if the material is not included under the Creative Commons license, users will need to obtain permission from the license holder to reproduce the material. To view a copy of this license, visit http://creativecommons.org/licenses/by/4.0/ 